\title{
Left atrial strain - a current clinical perspective
}

\author{
Karolina Kupczyńska', Giulia Elena Mandoli², Matteo Cameli², Jarosław D Kasprzak \\ 119t Department and Chair of Cardiology, Bieganski Hospital, Medical University, Łódź, Poland \\ 2 Department of Medical Biotechnologies, Division of Cardiology, University of Siena, Siena, Italy
}

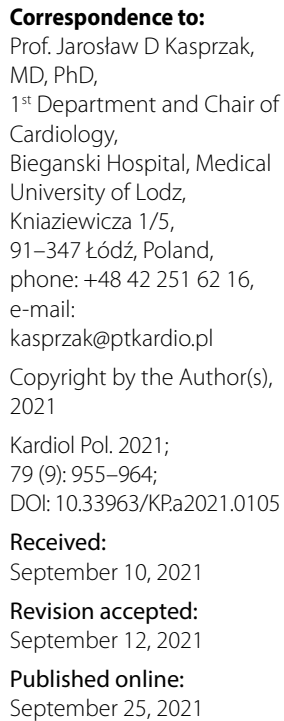

\section{FUNCTIONAL SIGNIFICANCE OF THE LEFT ATRIUM}

Dysfunction and structural remodeling of the left atrium (LA) are significant components of cardiovascular pathology [1,2]. Early detection of these abnormalities contributes to evaluating even preclinical diagnosis of ventricular dysfunction or valve disease. It can also corroborate the diagnosis of emerging atrial cardiomyopathy $[3,4]$.

Traditional echocardiographic evaluation of the LA used to be oversimplified and limited to the anteroposterior dimension and semi quantification of atrial component of ventricular filling, and only recently it was supplemented with standardized volumetry [5]. However, routine measures of atrial function have not been implemented yet [6].

LA function consists of three components, mainly: reservoir, conduit, and active pump [7]. In the case of normal diastolic function, the relative contribution of the particular LA phases into the left ventricular (LV) filling is as follows: reservoir $40 \%$, conduit 35\%, pump $25 \%$ [8].

LA contractility modulates LV filling and plays an essential role in maintaining cardiac output even in the setting of impaired relaxation or reduced compliance of $\operatorname{LV}[1,7]$. The importance of reservoir and pump phases increases, and the conduit phase's role decreases in patients with disturbed LV relaxation [8]. The impaired phasic function of the LA was described in many cardiovascular diseases, including atrial fibrillation (AF), stroke, cardiomyopathies, and valvular heart disease [2].

\section{LEFT ATRIAL STRAIN ASSESSMENT}

Speckle-tracking echocardiography (STE) has become an established method for quantifying myocardial function [9]. It provides reliable estimates of myocardial deformation with further accuracy, thanks to the recent advent of dedicated software packages. Measurements of atrial strain using STE are well-validated [10,11] and, notably, correlated with the histologically proven fibrotic remodeling of the LA wall [12-14]. However, the results obtained with different echocardiographic machines and post-processing software packages should be interpreted with caution because vendor variability is not well studied yet.

In contrast to Doppler techniques, STE is independent of the angle of the ultrasound beam and cardiac translational movements. A two-dimensional speckle consisting of a group of pixels is traced in all directions within the imaging plane. The main limitation can be partial displacement of the speckles outside the imaging plane. Three-dimensional echocardiography may overcome this limitation by analysis within a volumetric dataset [15]. The most frequently used measurement based on the STE technique 
is global longitudinal strain, describing the relative change in the muscle length averaged for visible segments [5]. The strain is a change in the distance between two points of the myocardium occurring in the cardiac cycle. When the distance decreases (shortening), the deformation has a negative value, and when the distance increases (lengthening), its value is positive. The strain rate reflects the rate of change of the mentioned distance $[16,17]$.

The majority of published studies on the LA strain (LAS) are based on the measurements performed with LV-dedicated software, but several dedicated tools are currently available. In addition, due to increasing interest in the functional assessment of the LA, the international expert group has recently published a document to standardize LAS evaluation [6].

LAS is measured from the apical 4- and 2-chamber views. For a reliable analysis, the apical views should be optimized to avoid foreshortening and to record a maximized cross-sectional view of the LA cavity. The assessment can involve only a 4-chamber view (6 LA segments) or both 4- and 2-chamber views to report the average value from 12 segments (Figure 1). The 3-chamber view should not be taken into account because of the proximity of the aortic valve and aorta, which could falsify LAS measurements $[2,6]$. It is crucial to keep the frame rate above $60[18,19]$.

LAS measurements should be interpreted with caution for the definition of the reference point. In fact, for LAS, two possibilities were explored, using QRS-complex or P-wave as the starting points for LA border detection [16, $17,20]$. The software automatically indicates the upslope of the R-wave as the starting point and generates the frame for endocardial tracing. This timepoint is a surrogate for end-diastole [6]. The analysis, which is focused on other zero-reference points, such as the onset of QRS complex or P-wave, requires additional manipulations on the ECG curve [10]. The strain curve with different starting points is

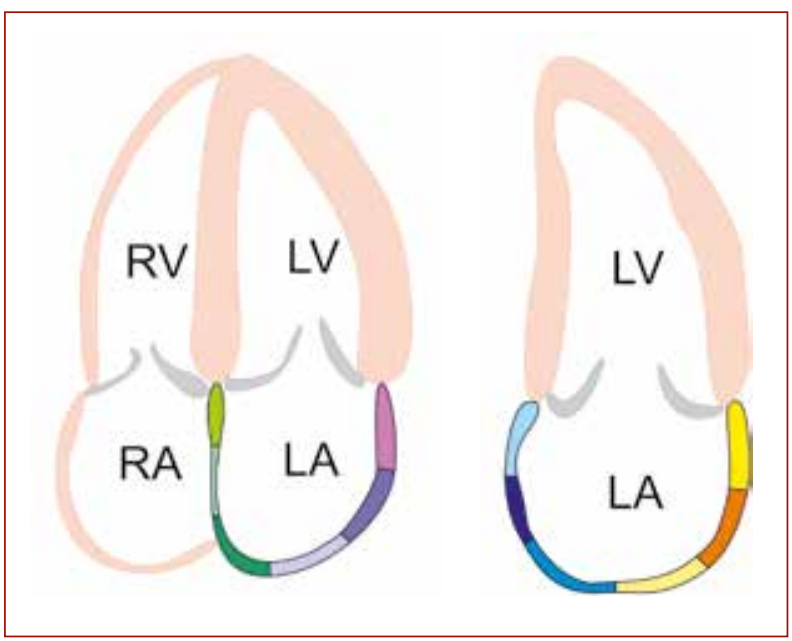

Figure 1. Segmental division of the left atrium in 4- and 2-chamber apical views using the speckle tracking echocardiography

Abbreviations: LA, left atrium; LV, left ventricle; RA, right atrium; RV, right ventricle presented in Figure 2. Data from 26 expert centers (MASCOT HIT study, Multicentric Atrial Strain COmparison between Two different modalities) proved that both methods are characterized by similar reproducibility. Still, calculations using the QRS complex as a reference point were associated with better feasibility and shorter analysis time [10]. This convention is also more universal because it can be applied in AF. The strain curve generated using this method enables the assessment of:

- LA strain in the reservoir phase (LAS-r) corresponding to LA early diastole with maximum relaxation of its wall, also known as peak atrial longitudinal strain (PALS);

- LA strain in the conduit phase (LAS-cd) corresponding to LA mid-diastolic emptying with its passive shortening;

- LA strain in the contraction phase (LAS-ct) or peak atrial contraction strain (PACS) corresponding to LA systole with active myocardial shortening producing the atrial contribution to LV filling [21].

Thus, the total function of the LA is best reflected by reservoir strain expressed in percentage points, algebraically positive.

The LAS calculated using $\mathrm{P}$-wave as the starting point is known as epsilon $(\varepsilon)$. The generated strain curve allows to assess:

- peak negative $\varepsilon$ corresponding to LA contractile;

- peak positive $\varepsilon$ corresponding to LA conduit phase;

- total $\varepsilon$ as the absolute value of both abovementioned peaks, reflecting the LA reservoir phase [22].

Recently available software dedicated to the LA, such as AutoStrain LA or LA Automated Function Imaging, allows for a quick assessment with a smooth transition between the P-wave and R-wave methods. For example, Figure 3 shows the results of the LA strain assessment with AutoStrain LA.

STE also enables the assessment of the LA strain rate by a curve with three peaks. In the analysis with R-wave set as a reference point, the first peak is positive and corresponds to the LA reservoir phase. The consecutive two peaks are negative. The first corresponds to the LA conduit phase (passive LA strain rate) and the second one to the LA contractile (active LA strain rate) [23, 24]. However, STE strain rate analysis is of less interest in the literature. A significant limitation is the temporal resolution of acquired images. The frame rate should be increased significantly in tachycardia. The strain rate as a time-dependent parameter requires higher temporal resolution, optimally $>100$ frames per second [25]. A recently developed novel imaging technique enabling the acquisition of images with 200 frames per second is a promising prospect [26].

The analysis of LAS dispersion is possible due to advanced assessment of regional data and is calculated as the standard deviation of regional values of time to the maximum peak of segmental strain curves normalized to the R-R interval $[27,28]$. 

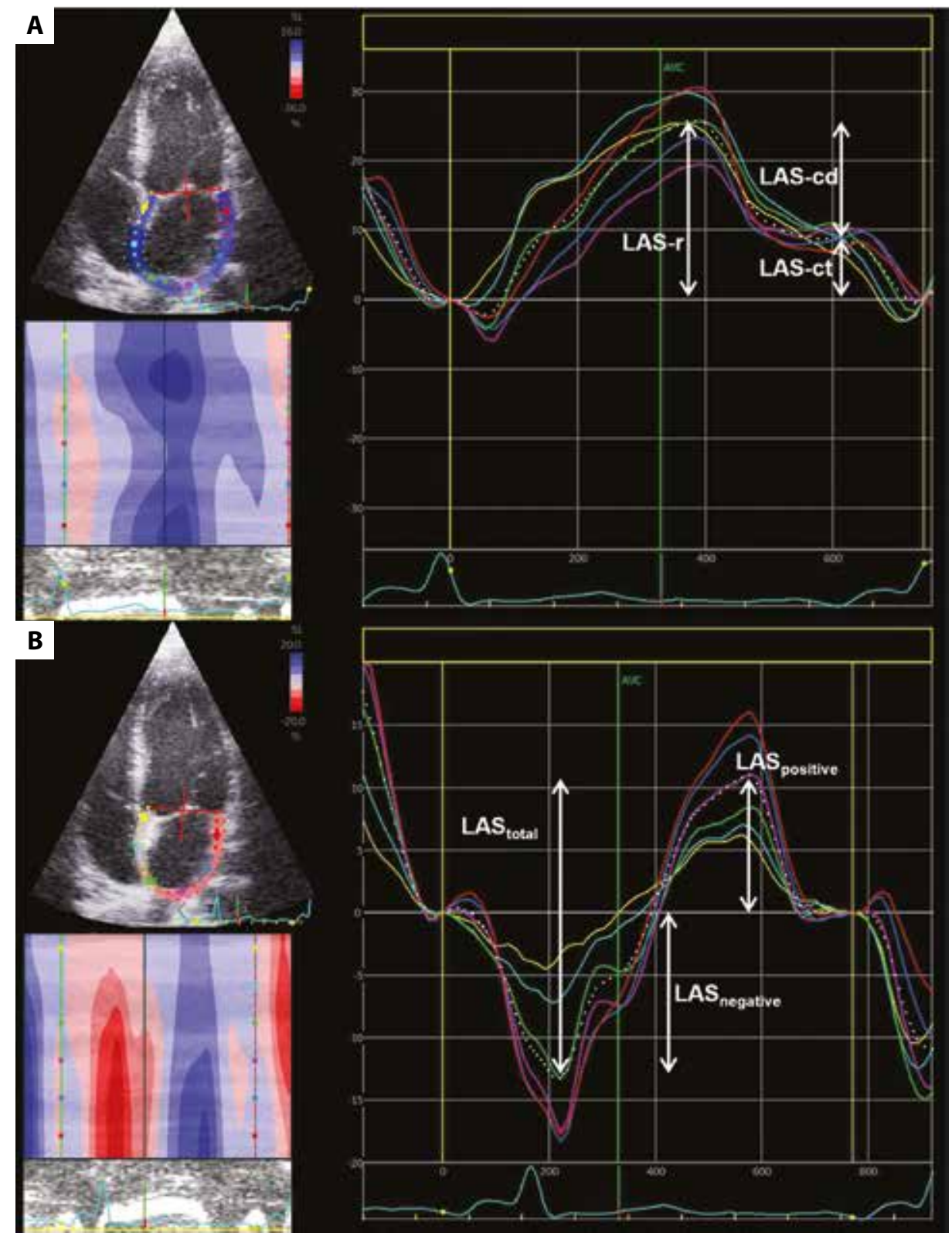

Figure 2. Left atrial strain (LAS) nomenclature depending on the choice of zero reference timepoint - QRS-complex (panel A) and P-wave (panel B). When $\mathrm{R}$-wave is set as the starting point, the first positive peak corresponds to the left atrium (LA) reservoir phase, the second peak characterizes LA contractile, and the difference between those peaks corresponds to the conduit phase. When P-wave is set as the reference, the first negative peak characterizes the LA contraction, positive peak - conduit phase, and their sum corresponds to the LA reservoir phase

Several parameters derived from LAS were proposed to describe the cardiac function better. The LA stiffness index reflecting decreased compliance of $L A$ can be non-invasively calculated as the quotient of mitral E/E' ratio and LAS-r. This parameter can be used as a surrogate of invasively measured stiffness requiring pulmonary capillary wedge pressure assessment. The LA stiffness index was revealed to be useful for differentiating healthy controls, patients with diastolic dysfunction, and patients with heart failure (HF) and preserved and reduced ejection fraction [29]. Moreover, this parameter is related to collagen synthesis and predicts AF recurrence after pulmonary vein isolation [30]. Interestingly, the LA stiffness index is decreased in competitive athletes coexisting with the enlarged LA as physiological adaptation [31].

LA function should be interpreted in the context of $\mathrm{LV}$, and it is interesting to analyze atrioventricular strain calculated as the sum of absolute LAS-r and LV strain.
However, in patients with hypertension and/or diabetes mellitus without alteration of standard echo parameters, the best marker of subclinical abnormalities was LAS-r [32].

Experience with LAS calculated from 3D datasets is preliminary, but current software used for 4D LA analysis automatically calculates the strain curve with longitudinal and circumferential strain values (Figure 4).

\section{NORMAL VALUES OF LEFT ATRIAL STRAIN}

A meta-analysis of 40 studies conducted predominantly using EchoPAC software (General Electric Healthcare, Milwaukee, WI, USA) defined a range of normal values for atrial deformation (Table 1). The authors compiled data obtained both with tools provided by General Electric and other suppliers, without noting significant vendor-related differences [33].

The authors of the multicenter NORRE study (Normal Reference Ranges for Echocardiography) determined 


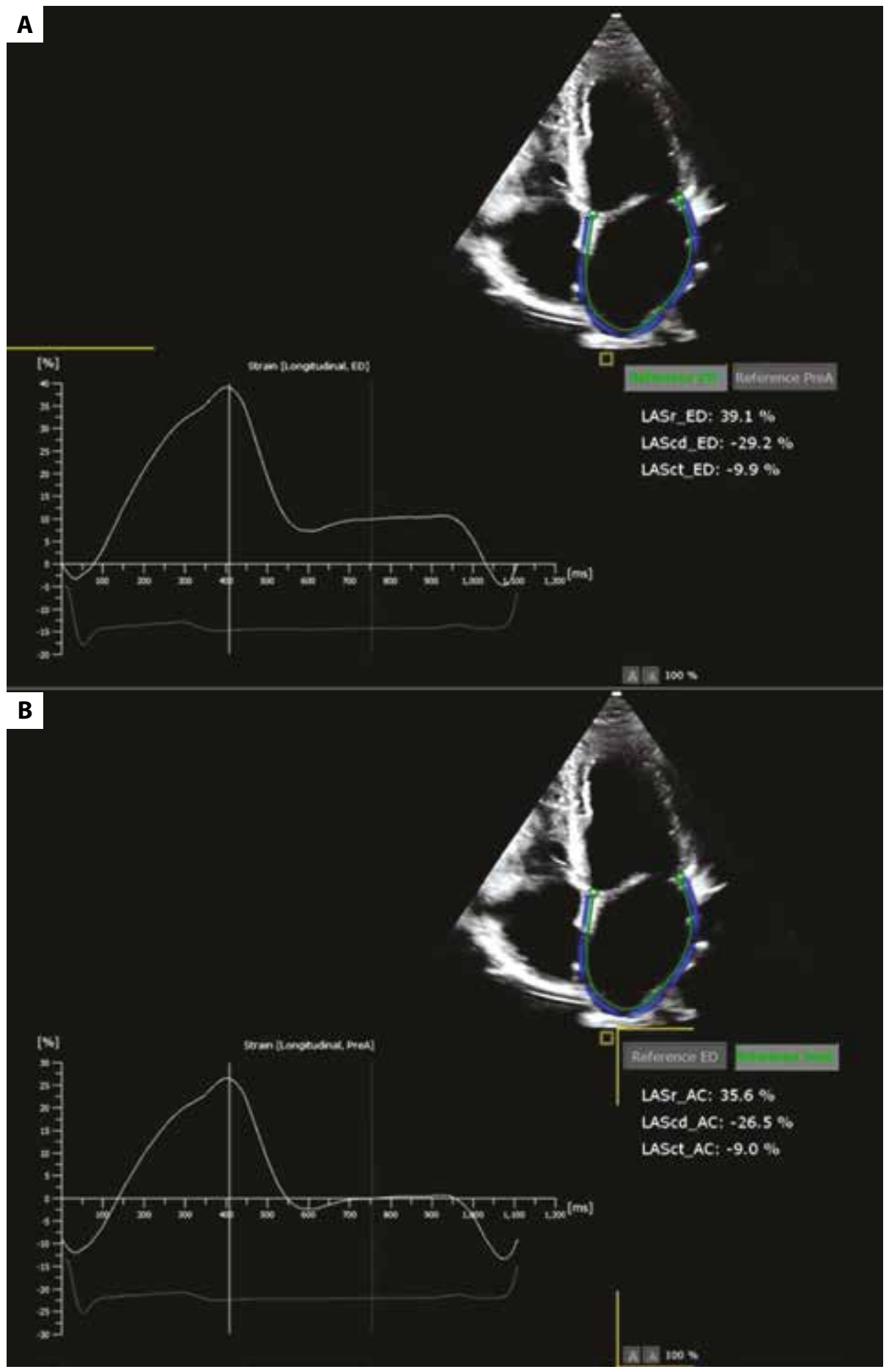

Figure 3. Left atrial strain derived left atrium (LA) dedicated software (AutoStrain LA). Strain curve generated depending on zero-reference timepoint - QRS-complex (panel A) and P-wave (panel B). Courtesy Philips Poland

a range of reference values for LAS and LA stiffness index using commercially available VIS — Vendor Independent Software (TomTec Imaging System, Unterschleißheim, Germany) (Table 2). The study included 371 healthy individuals. In the multivariate analysis, it turned out that age is independently related to the individual components of deformation, regardless of gender or an echocardiograph type used for registration [11].

Table 3 shows the strain values obtained in the MASCOT HIT study and derived from three study groups (healthy volunteers, patients with LV pressure overload, or LV volume overload). The median time needed for analysis with R-wave reference point was shorter (120 sec, interquartile range [IQR], 80-165 sec) than with P-wave set as a starting point (110 sec, IQR, 78-149 sec) [10].

\section{ATRIAL CARDIOMYOPATHY}

An important concept related to the LA is atrial cardiomyopathy, defined as a complex of changes in the structure, tissue architecture, contractility, or electrophysiological 


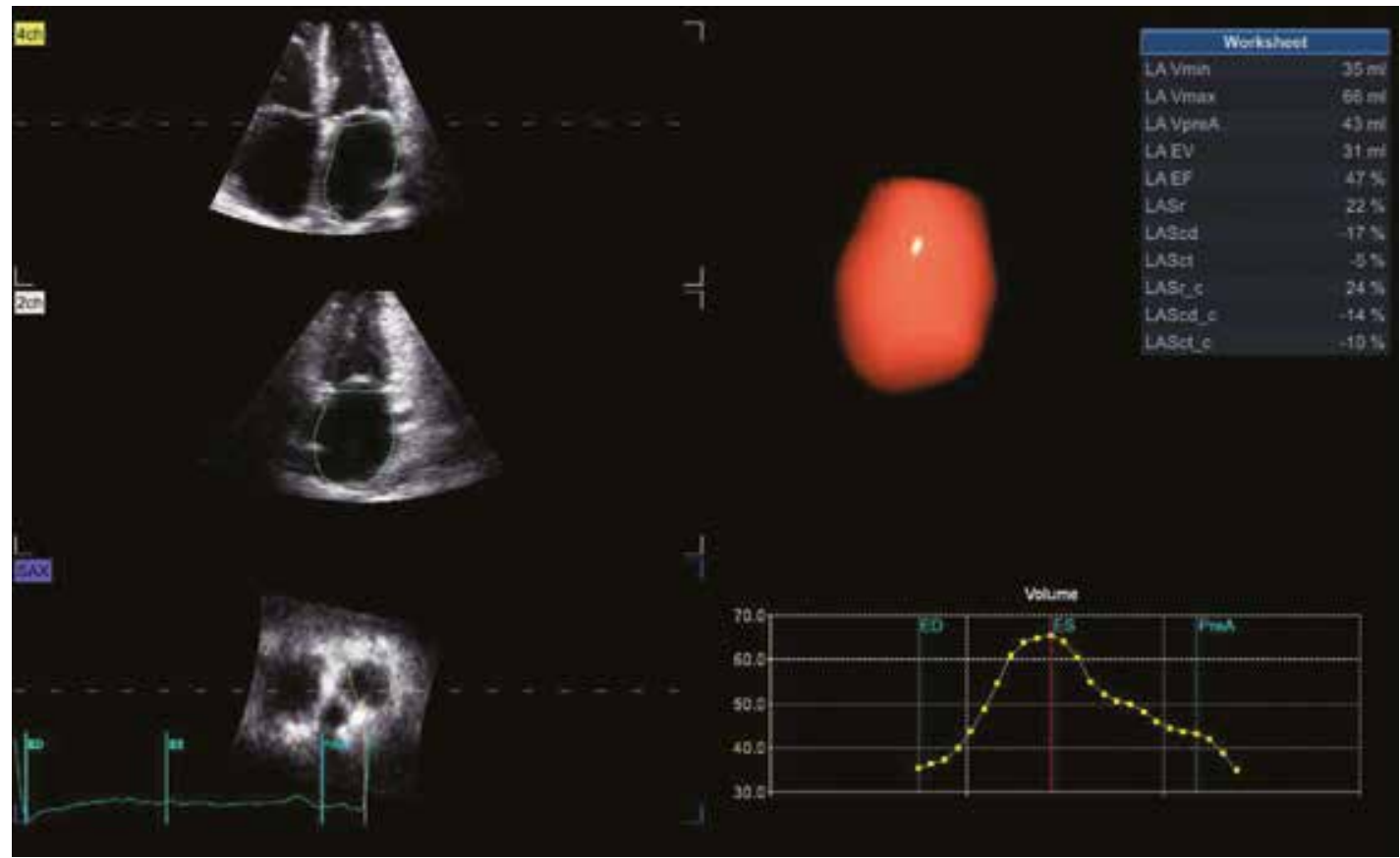

Figure 4. Left atrial assessment derived from 4D full volume data set enabling volumetric and strain analysis (Automated Function Assessment, Echopac, GE Healthcare, Chicago, IL, USA)

Abbreviations: LAScd, longitudinal strain during conduit phase; LASct, longitudinal strain during contraction phase; LASr, longitudinal strain during reservoir phase; LAScd_c, circumferential strain during conduit phase; LASct_c, circumferential strain during contraction phase; LAS$r_{-} c$, circumferential strain during reservoir phase

Table 1. Summary of the left atrial strain reference values obtained from the meta-analysis [33]

\begin{tabular}{l|c|c} 
& Comments \\
\hline LAS-r, \% & Mean $(\mathbf{9 5} \% \mathbf{C I})$ & 40 studies (2542 subjects) \\
LAS-ct, \% & $39.4(38-40.8)$ & 18 studies (1005 subjects) \\
LAS-cd, \% & $17.4(16-19)$ & 14 studies (805 subjects) \\
\hline
\end{tabular}

Abbreviations: LAS-cd, left atrial strain — phase of conduit; LAS-ct, left atrial strain — phase of contraction; LAS-r, left atrial strain — phase of reservoir

Table 2. Reference values defined in the NORRE study for the individual components of left atrial deformation (lower limit of normal) and the stiffness index (upper limit of normal) [11]48.7\%, and $41.4 \%$ for left atrial strain (LAS

\begin{tabular}{l|c|c|c} 
Age range & $\begin{array}{c}\mathbf{2 0 - 4 0} \text { years } \\
(\mathbf{n}=\mathbf{1 3 7})\end{array}$ & $\begin{array}{c}\mathbf{4 0 - 6 0} \text { years } \\
(\mathbf{n}=\mathbf{1 7 3})\end{array}$ & $\begin{array}{c}\mathbf{7 6 0} \text { years } \\
(\mathbf{n}=\mathbf{6 1})\end{array}$ \\
LAS-r, \% & $\geq 31.1$ & $\geq 27.7$ & $\geq 22.7$ \\
LAS-ct, \% & $\geq 7.2$ & $\geq 9.3$ & $\geq 7.7$ \\
LAS-cd, \% & $\geq 16.2$ & $\geq 12$ & $\leq 11.5$ \\
LA stiffness index & $\leq 0.22$ & $\leq 0.42$ & $\leq 0.55$ \\
\hline
\end{tabular}

Abbreviations: LA stiffness index — ratio of E/E' to LAS-r (dimensionless parameter). Other — see Figure 1 and Table 1

Table 3. Summary of left atrial strain values obtained in the MASCOT HIT study for all subgroups: healthy individuals, patients with pressure overload (hypertension or aortic stenosis), and patients with left ventricular volume overload (heart failure or mitral regurgitation) [10]

\begin{tabular}{l|c|c|c|c|c|c|} 
& \multicolumn{2}{|c|}{ Healthy volunteers } & \multicolumn{2}{c}{ LV pressure overload } & \multicolumn{2}{c}{ LV volume overload } \\
\hline & P-wave & R-wave & P-wave & R-wave & P-wave & R-wave \\
\hline LAS-r, $\%$ & $30.5 \pm 8$ & $33.5 \pm 10.9$ & $21.9 \pm 6.8$ & $23.0 \pm 8.5$ & $19.2 \pm 7.4$ \\
LAS-ct, $\%$ & Not published & $15 \pm 5.3$ & Not published & $13.4 \pm 5.7$ & Not published & $18.9 \pm 9.2$ \\
\hline
\end{tabular}

Abbreviations: see Figure 1 and Table 1

characteristics affecting the atria with the possibility of clinically significant manifestation [34]. LAS can be easily implemented to identify abnormalities in LA function corresponding to atrial fibrosis. Furthermore, STE detects LA dysfunction before anatomical changes occur [4,
35]. However, it should be emphasized that atrial cardiomyopathy is currently a pathophysiological concept rather than a clinical unit with specific diagnostic criteria and treatment rules, and further research is needed in this field. 


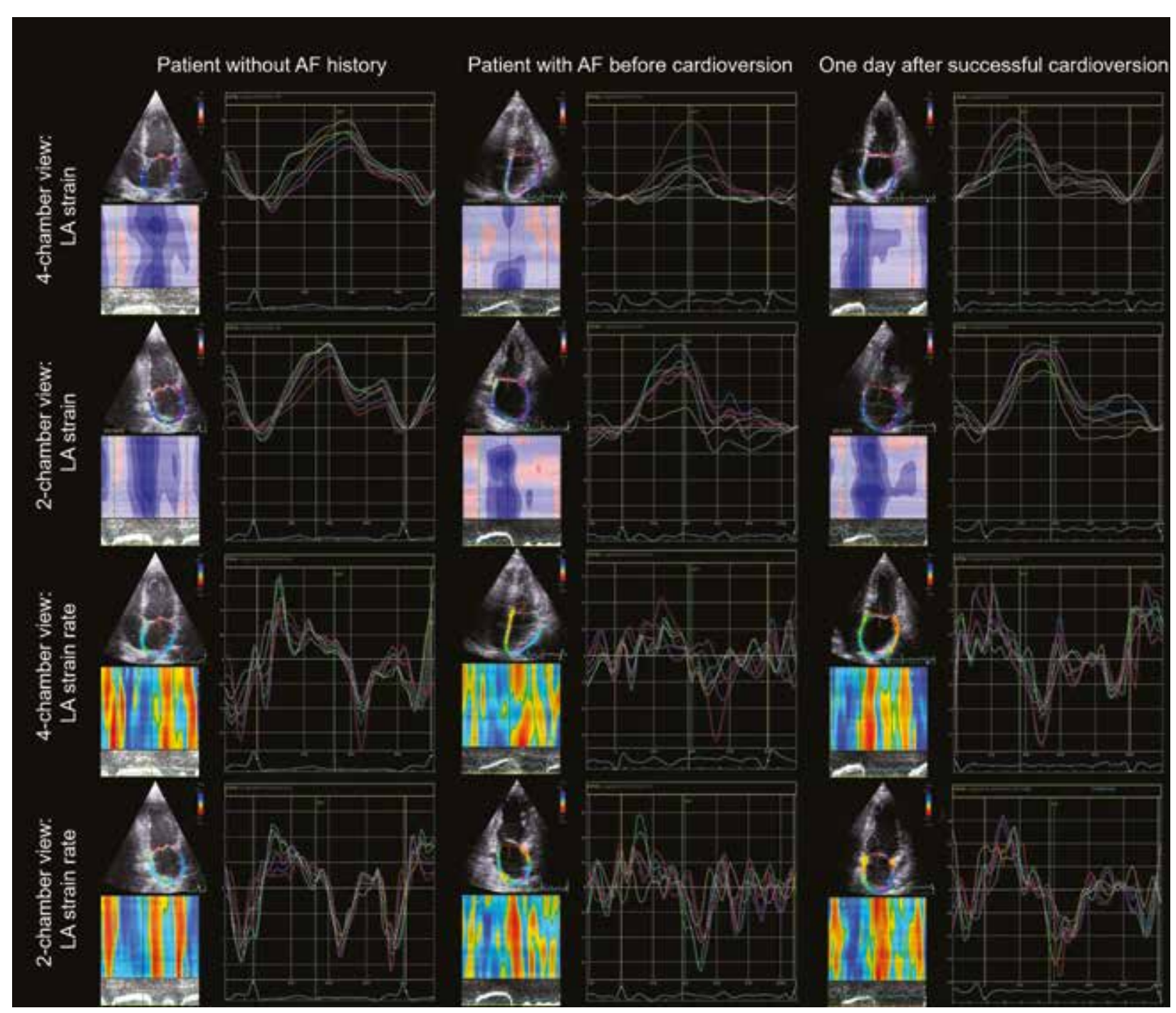

Figure 5. Left atrial strain and strain rate assessment in a patient without atrial fibrillation history (left panel), and in a patient with atrial fibrillation before and one day after successful electrical cardioversion (middle and right panels respectively)

\section{ATRIAL FIBRILLATION}

$\mathrm{AF}$ is a factor contributing to the development of atrial cardiomyopathy and its manifestation, which may explain the weak temporal relationship between episodes of AF and embolic stroke [36].

LAS both in the reservoir (optimal cut-off value LAS-r $\leq 19 \%$ ) and contractile phase (LAS-ct $\leq 8.7 \%$ ) enables the identification of patients with a history of AF in the population with hypertension [37].

Abnormal LAS is a proven predictor of AF occurrence, especially in patients after cryptogenic stroke [38, 39]. Recently, Kawakami et al. proposed an innovative approach, analyzing whether the assessment of LA strain brings additional benefits against well-established LV strain analysis. Based on the obtained results, the authors proposed an algorithm in which a patient with an increased 5-years risk of AF and normal LA volume should at first have LAS-ct assessed while in the case of increased LA volume, LV global longitudinal strain should be evaluated first [40].
Surprisingly few studies refer to LA deformation in the active phase of the pump as a prognostic factor [21]. However, this parameter assessed on the first day after successful electrical cardioversion showed a predictive role in maintaining sinus rhythm during a one-year follow-up. The value of LAS-ct $>3.4 \%$ derived from the 4-chamber view analysis was an optimal cut-off. LAS-r $>14.6 \%$ was a significant predictor of survival without AF recurrence [41]. Figure 5 shows the comparison of LA strain results in a patient without a history of AF and a patient with AF, before and after successful electrical cardioversion.

A LAS- $r \leq 10,75 \%$ had a sensitivity of $85 \%$ and a specificity of $99 \%$ in predicting 6-months AF recurrence after electrical cardioversion carried out due to persistent and long-lasting persistent AF and was an independent marker in multivariate analysis [42].

Impaired LA function favors the formation of thrombi, especially in the LA appendage. Intra-atrial thrombus was related to $L A$ dysfunction assessed during $A F$ with the best discriminating power by a LAS dispersion $>22 \%$ (sensitivity: 


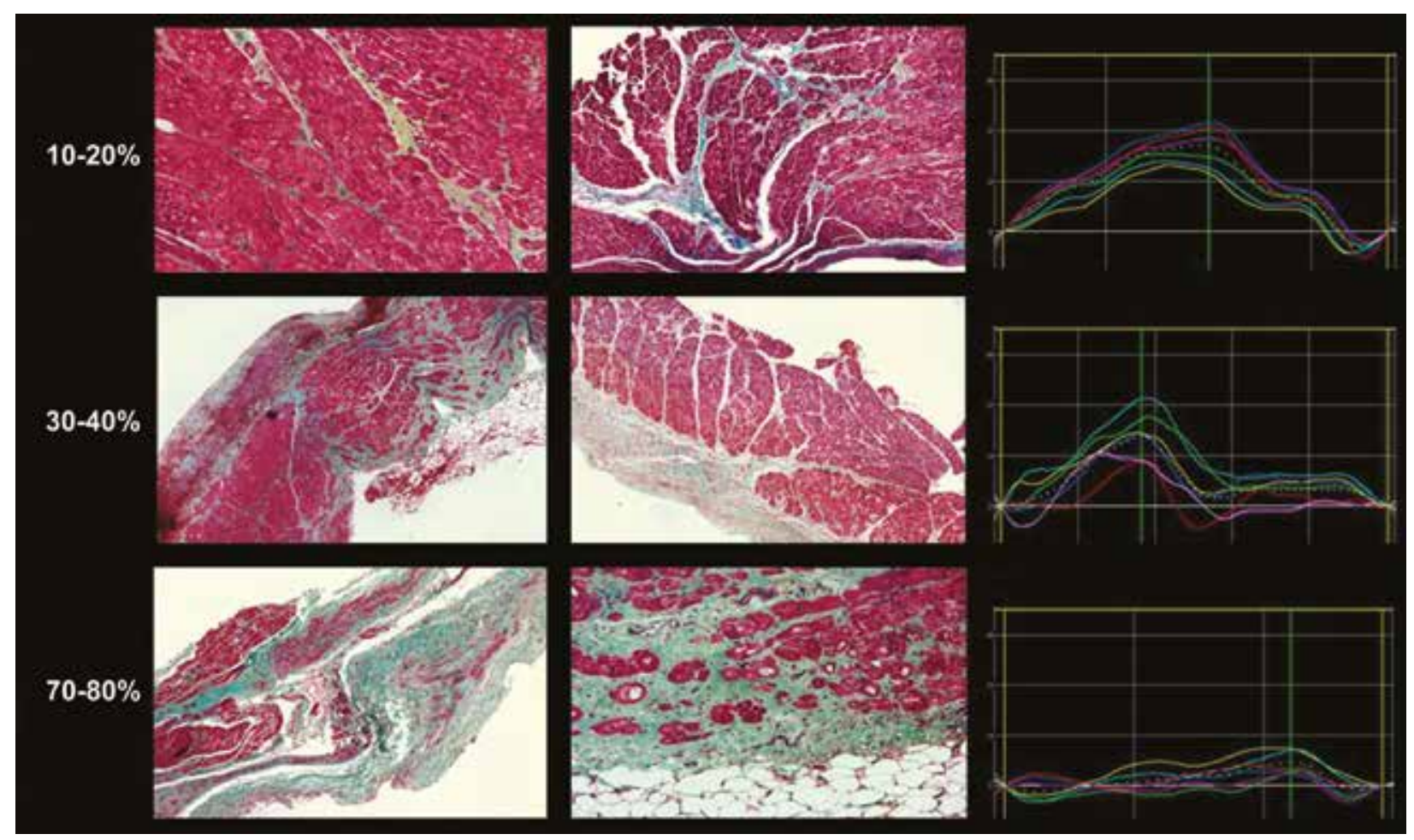

Figure 6. Left atrial strain correlates with fibrosis on atrial samples. Comparison between left atrial bioptic specimens (hematoxylin and eosin staining) with increasing amounts of wall myocardial fibrosis and corresponding left atrial strain curves, showing progressively reduced longitudinal deformation. Adapted from Lisi et al. [49]

45\% and specificity: $89 \%$ ) and LAS-r $\leq 11 \%$ (sensitivity: $74 \%$, specificity: 65\%) [27]. Moreover, both parameters were independent factors related to the presence of thrombi, even after adjustment for $\mathrm{CHA}_{2} \mathrm{DS}_{2}$-VASc score $[27,43]$, and LAS dispersion adjusted for $L V$ ejection fraction or LV global longitudinal strain [27].

Similarly, Obokata et al. reported that LAS-r assessed in AF improved the predictive ability of the $\mathrm{CHA}_{2} \mathrm{DS}_{2}-\mathrm{VASC}$ score in the prediction of systemic embolism and was also an independent prognostic factor of mortality following stroke. The best criterion for discriminating between patients with stroke and a demographically and clinically matched control group with AF but without an embolic event was LAS- $r<15.4 \%$. In this study, LA roof segments were excluded from the analysis [44].

\section{HEART FAILURE}

The importance of LA assessment is demonstrated by including its enlargement as one of the diagnostic criteria in the current HF definition [45]. In addition to its prognostic significance, LA volume is also used to assess LV diastolic function and the diagnosis of HF with preserved LV ejection fraction (HFpEF) $[46,47]$. However, LA function could be already abnormal even if LA size is preserved.

Patients with HFpEF had impaired LAS-r and LA passive and active strain rates compared to healthy controls. Moreover, HFpEF patients with LA enlargement or a history of AF had lower LA strain values [24].

Another study revealed in a group of 4312 consecutive patients with acute HF that LAS- $r<18 \%$ identifies individu- als who may develop new-onset AF within 5-year follow-up, both in the HF with reduced ejection fraction (HFrEF) subgroup and analyzed together HFpEF and HF with mid-range ejection fraction (HFmrEF) [48]. In advanced HFrEF, LAS-r strongly correlated with the amount of invasively analyzed fibrosis on LA bioptic samples (Figure 6) [49].

\section{DIASTOLIC DYSFUNCTION}

The quantification of LA volume is used to support the diagnosis of LV diastolic function. However, macroscopic changes in its structure are the late manifestation of dysfunction. Left atrial strain deteriorates along with the severity of diastolic dysfunction and is proposed as a helpful parameter to distinguish particular grades of diastolic dysfunction with good to excellent diagnostic ability. A LAS-r $>35 \%$ allowed to differentiate patients with normal diastolic function with an accuracy of $72 \%$. Moreover, a LAS-r $\leq 19 \%$ had the accuracy of $95 \%$ to identify patients with the $3^{\text {rd }}$ grade of diastolic dysfunction [50]. The cut-off of LAS-r $<20 \%$ was proposed to improve the diagnosis in case of an indeterminate degree of diastolic dysfunction [51].

\section{CORONARY ARTERY DISEASE}

The LA can also be affected by ischemic heart disease, mainly as a consequence of LV myocardial damage. Both LAS-r and LAS-ct were impaired in patients with acute myocardial infarction, with a circumflex artery identified as a culprit lesion [52]. LAS-r assessed within 48 hours after primary percutaneous coronary intervention for the first ST-segment elevation acute myocardial infarction was 
the independent predictor associated with a lower risk of adverse LV remodeling (OR, 0.77; $P=0.003$; optimal cutoff, $28.9 \%)$ and adverse clinical events (OR, 0.88; $P=0.04$; optimal cut-off, 23.8\%) during 6-month follow-up [53].

\section{CARDIAC AMYLOIDOSIS}

Cardiac amyloidosis, in the study by Nochioka et al., was associated with severe LA dysfunction. Mean LAS-r was significantly lower in the patients with amyloidosis than in healthy controls $(18.8 \pm 11.6 \%$ vs $40.6 \pm 6.2 \% ; P<0.001)$. It should be mentioned that differences in LA function were identified between amyloid subtypes with most abnormal LAS-r in wild-type transthyretin amyloidosis [54].

\section{VALVULAR HEART DISEASE}

LA functional parameters decrease in valvular heart disease and have prognostic significance. In asymptomatic patients with pure mitral valve stenosis, LAS- $r<17.4 \%$ showed a good predictive value to predict $A F$ in 4-year follow-up [55]. In the study by Sugimoto et al., LA pump and reservoir function were impaired in patients with primary and secondary mitral regurgitation. The subgroup with primary, but not secondary, regurgitation had some exercise reserve in LA functional parameters, even in moderate to severe regurgitation. Patients with mild to moderate secondary mitral regurgitation had a higher stiffness index than patients with moderate to severe primary mitral regurgitation during exercise. Moreover, LAS- $r>16 \%$ at peak exercise predicted a 3-year event-free survival [56]. A reduced basal LAS-r could also predict mid-term adverse events, including $A F$, stroke, acute $H F$, and cardiovascular death, in patients with asymptomatic moderate mitral regurgitation, helping identify a subpopulation of patients with a more advanced stage of the disease [57].

STE analysis of LA had very good predictive value for the prognosis of postoperative AF in patients with aortic stenosis undergoing surgical replacement. The LAS-r $<16.9 \%$ had a sensitivity of $86 \%$ and specificity of $91 \%$ in predicting new-onset AF after implantation of biological aortic valve prosthesis [58].

\section{ANTHRACYCLINE THERAPY}

Recently published results proved the importance of LA strain assessment in monitoring the cardiotoxic effects of cancer chemotherapy. STE analysis enables early diagnosis of cardiac dysfunction and starting treatment. Significant reduction in LAS-r can be defined as a relative decrease of $>10 \%$ or LAS-r $<35 \%$. LAS-r and LAS-cd, but not LAS-ct, showed a significant decline in patients with breast cancer during doxorubicin therapy [59].

\section{CONCLUSIONS}

The LA function assessment by STE is a relatively simple and robust tool allowing to disclose pathophysiological mechanisms in the broad spectrum of cardiovascular disease. The prognostic significance of LA strain has been demonstrated in many clinical scenarios, including the definition of diastolic dysfunction, cardioembolic risk, and even cardiovascular mortality in a selected patient population. The most actual diagnostic application includes heart failure and atrial cardiomyopathy (with emphasis on identifying AF episodes) and may influence clinical decision-making, refining the prediction of rhythm-control strategy success in AF.

\section{Article information}

Conflicts of interest: None declared.

Open access: This article is available in open access under Creative Common Attribution-Non-Commercial-No Derivatives 4.0 International (CC BY-NC-ND 4.0) license, allowing to download articles and share them with others as long as they credit the authors and the publisher, but without permission to change them in any way or use them commercially. For commercial use, please contact the journal office at kardiologiapolska@ptkardio.pl.

How to cite: Kupczyńska K, Mandoli GE, Cameli M, Kasprzak JD. Left atrial strain - a current clinical perspective. Kardiol Pol. 2021; 79(9): 955-964, doi: 10.33963/KP.a2021.0105.

\section{REFERENCES}

1. Rosca M, Lancellotti P, Popescu BA, et al. Left atrial function: pathophysiology, echocardiographic assessment, and clinical applications. Heart. 2011; 97(23): 1982-1989, doi: 10.1136/heartjnl-2011-300069, indexed in Pubmed: 22058287.

2. Thomas L, Muraru D, Popescu BA, et al. Evaluation of left atrial size and function: relevance for clinical practice. J Am Soc Echocardiogr. 2020; 33(8): 934-952, doi: 10.1016/j.echo.2020.03.021, indexed in Pubmed: 32762920.

3. Donal E, Lip GYH, Galderisi M, et al. EACVI/EHRA expert consensus document on the role of multi-modality imaging for the evaluation of patients with atrial fibrillation. Eur Heart J Cardiovasc Imaging. 2016; 17(4): 355-383, doi: 10.1093/ehjci/jev354, indexed in Pubmed: 26864186.

4. Hirsh BJ, Copeland-Halperin RS, Halperin JL. Fibrotic atrial cardiomyopathy, atrial fibrillation, and thromboembolism: mechanistic links and clinical inferences. J Am Coll Cardiol. 2015; 65(20): 2239-2251, doi: 10.1016/j. jacc.2015.03.557, indexed in Pubmed: 25998669.

5. Lang RM, Badano LP, Mor-Avi V, et al. Recommendations for cardiac chamber quantification by echocardiography in adults: an update from the American Society of Echocardiography and the European Association of Cardiovascular Imaging. J Am Soc Echocardiogr. 2015; 28(1): 1-39.e14, doi: 10.1016/j.echo.2014.10.003, indexed in Pubmed: 25559473.

6. Badano LP, Kolias TJ, Muraru D, et al. Standardization of left atrial, right ventricular, and right atrial deformation imaging using two-dimensional speckle tracking echocardiography: a consensus document of the EAC$\mathrm{VI} / \mathrm{ASE} /$ Industry Task Force to standardize deformation imaging. Eur Heart J Cardiovasc Imaging. 2018; 19(6): 591-600, doi: 10.1093/ehjci/jey042, indexed in Pubmed: 29596561.

7. Matsuda $\mathrm{Y}$, Toma $\mathrm{Y}$, Ogawa $\mathrm{H}$, et al. Importance of left atrial function in patients with myocardial infarction. Circulation. 1983;67(3):566-571, doi: 10.1161/01.cir.67.3.566, indexed in Pubmed: 6821898.

8. Callegari S, Macchi E, Monaco R, et al. Myocyte changes and their left atrial distribution in patients with chronic atrial fibrillation related to mitral valve disease. Hum Pathol. 2005; 36(10): 1080-1089, doi: 10.1016/j. humpath.2005.07.018, indexed in Pubmed: 16226107.

9. Szymczyk E, Lipiec P, Michalski B, et al. 2D and 3D acoustic marker tracking technique: clinical application [article in Polish]. Kardiol Pol. 2013; 71(1): 77-83.

10. Cameli M, Miglioranza MH, Magne J, et al. Multicentric atrial strain comparison between two different modalities: MASCOT HIT study. Diagnostics (Basel). 2020; 10(11), doi: 10.3390/diagnostics10110946, indexed in Pubmed: 33202837.

11. Sugimoto T, Robinet $S$, Dulgheru $R$, et al. NORRE Study. Echocardiographic reference ranges for normal left atrial function parameters: results from the EACVI NORRE study. Eur Heart J Cardiovasc Imaging. 2018; 19(6): 630-638, doi: 10.1093/ehjci/jey018, indexed in Pubmed: 29529180. 
12. Cameli M, Lisi M, Focardi M, et al. Left atrial deformation analysis by speckle tracking echocardiography for prediction of cardiovascular outcomes. Am J Cardiol. 2012; 110(2): 264-269, doi: 10.1016/j.amjcard.2012.03.022, indexed in Pubmed: 22497676.

13. Mandoli GE, D'Ascenzi F, Vinco G, et al. Novel approaches in cardiac imaging for non-invasive assessment of left heart myocardial fibrosis. Front Cardiovasc Med. 2021; 8:614235, doi: 10.3389/fcvm.2021.614235, indexed in Pubmed: 33937354.

14. Her AY, Choi EY, Shim CY, et al. Prediction of left atrial fibrosis with speckle tracking echocardiography in mitral valve disease: a comparative study with histopathology. Korean Circ J. 2012; 42(5): 311-318, doi: 10.4070/kcj.2012.42.5.311, indexed in Pubmed: 22701133.

15. Mor-Avi V, Lang RM, Badano LP, et al. Current and evolving echocardiographic techniques for the quantitative evaluation of cardiac mechanics: ASE/EAE consensus statement on methodology and indications endorsed by the Japanese Society of Echocardiography. J Am Soc Echocardiogr. 2011; 24(3): 277-313, doi: 10.1016/j.echo.2011.01.015, indexed in Pubmed: 21338865 .

16. Hoit BD. Left atrial size and function: role in prognosis. J Am Coll Cardiol. 2014;63(6):493-505, doi: 10.1016/j.jacc.2013.10.055, indexed in Pubmed: 24291276.

17. Rimbaş RC, Mihăilă $S$, Vinereanu D. Sources of variation in assessing left atrial functions by $2 \mathrm{D}$ speckle-tracking echocardiography. Heart Vessels. 2016; 31(3): 370-381, doi: 10.1007/s00380-014-0602-8, indexed in Pubmed: 25388354.

18. Cameli M, Lisi M, Righini FM, et al. Novel echocardiographic techniques to assess left atrial size, anatomy and function. Cardiovasc Ultrasound. 2012; 10: 4, doi: 10.1186/1476-7120-10-4, indexed in Pubmed: 22296702.

19. Cameli $M$, Caputo $M$, Mondillo $S$, et al. Feasibility and reference values of left atrial longitudinal strain imaging by two-dimensional speckle tracking. Cardiovasc Ultrasound. 2009; 7: 6, doi: 10.1186/1476-7120-7-6, indexed in Pubmed: 19200402.

20. Hayashi S, Yamada H, Bando M, et al. Optimal analysis of left atrial strain by speckle tracking echocardiography: P-wave versus R-wave trigger. Echocardiography. 2015; 32(8): 1241-1249, doi: 10.1111/echo.12834, indexed in Pubmed: 25363348.

21. Pastore M, Mandoli G, Santoro C, et al. Left atrial strain in cardiovascular diseases : An overview of clinical applications. Cardiologia Hungarica. 2021; 51(1): 11-17, doi: 10.26430/chungarica.2021.51.1.11.

22. Saraiva RM, Demirkol S, Buakhamsri A, et al. Left atrial strain measured by two-dimensional speckle tracking represents a new tool to evaluate left atrial function.J Am Soc Echocardiogr. 2010;23(2): 172-180, doi: 10.1016/j. echo.2009.11.003, indexed in Pubmed: 20152699.

23. Miśkowiec D, Kupczyńska K, Michalski BW, et al. Left atrial dysfunction assessed by two-dimensional speckle tracking echocardiography in patients with impaired left ventricular ejection fraction and sleep-disordered breathing. Echocardiography. 2016; 33(1): 38-45, doi: 10.1111/echo.12987, indexed in Pubmed: 26058894.

24. Santos ABS, Kraigher-Krainer E, Gupta DK, et al. Impaired left atrial function in heart failure with preserved ejection fraction. Eur J Heart Fail. 2014; 16(10): 1096-1103, doi: 10.1002/ejhf.147, indexed in Pubmed: 25138249.

25. Voigt JU, Pedrizzetti G, Lysyansky P, et al. Definitions for a common standard for 2D speckle tracking echocardiography: consensus document of the EACVI/ASE/Industry Task Force to standardize deformation imaging. Eur Heart J Cardiovasc Imaging. 2015; 16(1): 1-11, doi: 10.1093/ehjci/jeu184 indexed in Pubmed: 25525063.

26. Fujikura K, Makkiya M, Farooq $M$, et al. Speckle-Tracking echocardiography with novel imaging technique of higher frame rate. J Clin Med. 2021; 10(10), doi: 10.3390/jcm10102095, indexed in Pubmed: 34068134.

27. Kupczynska K, Michalski BW, Miskowiec D, et al. Incremental value of left atrial mechanical dispersion over CHA DS -VASc score in predicting risk of thrombus formation. Echocardiography. 2018; 35(5): 651-660, doi: 10.1111/echo.13899, indexed in Pubmed: 29691894.

28. Dell'Era G, Rondano E, Franchi E, et al. Atrial asynchrony and function before and after electrical cardioversion for persistent atrial fibrillation. Eur JEchocardiogr. 2010; 11(7):577-583, doi: 10.1093/ejechocard/jeq010, indexed in Pubmed: 20400765.

29. Kurt M, Wang J, Torre-Amione $G$, et al. Left atrial function in diastolic heart failure. Circ Cardiovasc Imaging. 2009; 2(1): 10-15, doi: 10.1161/CIRCIMAGING.108.813071, indexed in Pubmed: 19808559.
30. Machino-Ohtsuka T, Seo Y, Tada H, et al. Left atrial stiffness relates to left ventricular diastolic dysfunction and recurrence after pulmonary vein isolation for atrial fibrillation. J Cardiovasc Electrophysiol. 2011; 22(9): 999-1006, doi: 10.1111/j.1540-8167.2011.02049.x, indexed in Pubmed: 21457382.

31. D'Ascenzi F, Pelliccia A, Natali BM, et al. Increased left atrial size is associated with reduced atrial stiffness and preserved reservoir function in athlete's heart. Int J Cardiovasc Imaging. 2015; 31(4): 699-705, doi: 10.1007/s10554-015-0600-7, indexed in Pubmed: 25627780.

32. Cameli M, Mandoli GE, Lisi E, et al. Left atrial, ventricular and atrio-ventricular strain in patients with subclinical heart dysfunction. Int J Cardiovasc Imaging. 2019; 35(2): 249-258, doi: 10.1007/s10554-018-1461-7, indexed in Pubmed: 30251175.

33. Pathan F, D'Elia N, Nolan MT, et al. Normal ranges of left atrial strain by speckle-tracking echocardiography: a systematic review and meta-analysis. J Am Soc Echocardiogr. 2017; 30(1): 59-70.e8, doi: 10.1016/j. echo.2016.09.007, indexed in Pubmed: 28341032.

34. Goette A, Kalman JM, Aguinaga L, et al. Document Reviewers:. EHRA/HRS/APHRS/SOLAECE expert consensus on atrial cardiomyopathies: Definition, characterization, and clinical implication. Heart Rhythm. 2017; 14(1): e3-e40, doi: 10.1016/j.hrthm.2016.05.028, indexed in Pubmed: 27320515.

35. Yuda S, Muranaka A, Miura T. Clinical implications of left atrial function assessed by speckle tracking echocardiography. J Echocardiogr. 2016; 14(3): 104-112, doi: 10.1007/s12574-016-0283-7, indexed in Pubmed: 26951561.

36. Healey JS, Brambatti M, Gold MR, et al. Temporal relationship between subclinical atrial fibrillation and embolic events. Circulation. 2014; 129(21): 2094-2099, doi: 10.1161/CIRCULATIONAHA.113.007825, indexed in Pubmed: 24633881

37. Petre I, Onciul S, lancovici $S$, et al. Left atrial strain for predicting atrial fibrillation onset in hypertensive patients. High Blood Press Cardiovasc Prev. 2019; 26(4): 331-337, doi: 10.1007/s40292-019-00326-4, indexed in Pubmed: 31309456.

38. Deferm S, Bertrand PB, Churchill TW, et al. Left atrial mechanics assessed early during hospitalization for cryptogenic stroke are associated with occult atrial fibrillation: a speckle-tracking strain echocardiography study. J Am Soc Echocardiogr. 2021; 34(2): 156-165, doi: 10.1016/j. echo.2020.09.009, indexed in Pubmed: 33132019.

39. Sade LE, Keskin S, Can U, et al. Left atrial mechanics for secondary prevention from embolic stroke of undetermined source. Eur Heart J Cardiovasc Imaging. 2020 [Epub ahead of print], doi: 10.1093/ehjci/jeaa311, indexed in Pubmed: 33206942.

40. Kawakami H, Ramkumar S, Pathan F, et al. Use of echocardiography to stratify the risk of atrial fibrillation: comparison of left atrial and ventricular strain. Eur Heart J Cardiovasc Imaging. 2020; 21(4): 399-407, doi: 10.1093/ehjci/jez240, indexed in Pubmed: 31578558.

41. Wałek $P$, Grabowska $U$, Cieśla $E$, et al. Left atrial longitudinal strain in the contractile phase as a predictor of sinus rhythm maintenance after electrical cardioversion performed due to persistent atrial fibrillation. Kardiol Pol. 2021; 79(4): 458-460, doi: 10.33963/KP.15913, indexed in Pubmed: 33784038

42. Moreno-Ruiz LA, Madrid-Miller A, Martínez-Flores JE, et al. Left atrial longitudinal strain by speckle tracking as independent predictor of recurrence after electrical cardioversion in persistent and long standing persistent non-valvular atrial fibrillation. Int J Cardiovasc Imaging. 2019; 35(9): 1587-1596, doi: 10.1007/s10554-019-01597-7, indexed in Pubmed: 30993507.

43. Kupczynska K, Michalski BW, Miskowiec D, et al. Association between left atrial function assessed by speckle-tracking echocardiography and the presence of left atrial appendage thrombus in patients with atrial fibrillation. Anatol J Cardiol. 2017; 18(1): 15-22, doi: 10.14744/AnatolJCardiol.2017.7613, indexed in Pubmed: 28559531.

44. Obokata M, Negishi K, Kurosawa K, et al. Left atrial strain provides incremental value for embolism risk stratification over $\mathrm{CHA}_{2} \mathrm{DS}_{2}-\mathrm{VASC}$ score and indicates prognostic impact in patients with atrial fibrillation. J Am Soc Echocardiogr. 2014; 27(7): 709-716.e4, doi: 10.1016/j.echo.2014.03.010, indexed in Pubmed: 24767972

45. Seferovic PM, Ponikowski P, Anker SD, et al. 2016 ESC Guidelines for the diagnosis and treatment of acute and chronic heart failure: The Task Force for the diagnosis and treatment of acute and chronic heart failure 
of the European Society of Cardiology (ESC)Developed with the special contribution of the Heart Failure Association (HFA) of the ESC. Eur Heart J. 2016; 37(27): 2129-2200, doi: 10.1093/eurheartj/ehw128, indexed in Pubmed: 27206819.

46. Nagueh SF, Smiseth $\mathrm{OA}$, Appleton $\mathrm{CP}$, et al. Recommendations for the evaluation of left ventricular diastolic function by echocardiography: an update from the American Society of Echocardiography and the European Association of Cardiovascular Imaging. J Am Soc Echocardiogr. 2016; 29(4): 277-314, doi: 10.1016/j.echo.2016.01.011, indexed in Pubmed: 27037982.

47. Kapłon-Cieślicka A, Kupczyńska K, Dobrowolski P, et al. On the search for the right definition of heart failure with preserved ejection fraction. Cardiol J. 2020; 27(5): 449-468, doi: 10.5603/CJ.a2020.0124, indexed in Pubmed: 32986238.

48. Park JJ, Park JH, Hwang IC, et al. Left atrial strain as a predictor of new-onset atrial fibrillation in patients with heart failure. JACC Cardiovasc Imaging. 2020; 13(10): 2071-2081, doi: 10.1016/j.jcmg.2020.04.031, indexed in Pubmed: 32682715.

49. Lisi M, Mandoli GE, Cameli M, et al. Left atrial strain by speckle tracking predicts atrial fibrosis in patients undergoing heart transplantation. Eur Heart J Cardiovasc Imaging. 2021 [Epub ahead of print], doi: 10.1093/ehjci/jeab106, indexed in Pubmed: 34118154

50. Singh A, Addetia K, Maffessanti F, et al. LA strain for categorization of LVdiastolic dysfunction. JACC Cardiovasc Imaging. 2017; 10(7): 735-743, doi: 10.1016/j.jcmg.2016.08.014, indexed in Pubmed: 28017389.

51. Cameli M, Mandoli GE, Mondillo S. Left atrium: the last bulwark before overt heart failure. Heart Fail Rev. 2017; 22(1): 123-131, doi: 10.1007/s10741-016-9589-9, indexed in Pubmed: 27873135.

52. Said KM, Nassar Al, Fouad A, et al. Left atrial deformation analysis as a predictor of severity of coronary artery disease. Egypt Heart J. 2018; 70(4): 353-359, doi: 10.1016/j.ehj.2018.09.004, indexed in Pubmed: 30591754.
53. Chu AA, Wu T, Zhang Lu, et al. The prognostic value of left atrial and left ventricular strain in patients after ST-segment elevation myocardial infarction treated with primary percutaneous coronary intervention. Cardiol J. 2021; 28(5): 678-689, doi: 10.5603/CJ.a2020.0010, indexed in Pubmed: 32037499

54. Nochioka K, Quarta CC, Claggett B, et al. Left atrial structure and function in cardiac amyloidosis. Eur Heart J Cardiovasc Imaging. 2017; 18(10): 1128-1137, doi: 10.1093/ehjci/jex097, indexed in Pubmed: 28637305.

55. Ancona R, Comenale Pinto $S$, Caso $P$, et al. Two-dimensional atrial systolic strain imaging predicts atrial fibrillation at 4-year follow-up in asymptomatic rheumatic mitral stenosis. J Am Soc Echocardiogr. 2013; 26(3): 270-277, doi: 10.1016/j.echo.2012.11.016, indexed in Pubmed: 23261148.

56. Sugimoto $T$, Bandera $F$, Generati $G$, et al. Left atrial dynamics during exercise in mitral regurgitation of primary and secondary origin: pathophysiological insights by exercise echocardiography combined with gas exchange analysis. JACC Cardiovasc Imaging. 2020; 13(1 Pt 1): 25-40, doi: 10.1016/j.jcmg.2018.12.031, indexed in Pubmed: 30878440.

57. Mandoli GE, Pastore MC, Benfari G, et al. Left atrial strain as a pre-operative prognostic marker for patients with severe mitral regurgitation. Int J Cardiol. 2021; 324: 139-145, doi: 10.1016/j.jicard.2020.09.009, indexed in Pubmed: 32920069.

58. Cameli M, Lisi M, Reccia R, et al. Pre-operative left atrial strain predicts post-operative atrial fibrillation in patients undergoing aortic valve replacement for aortic stenosis. Int J Cardiovasc Imaging. 2014; 30(2): 279-286, doi: 10.1007/s10554-013-0323-6, indexed in Pubmed: 24202403.

59. Laufer-Perl M, Arias O, Dorfman SS, et al. Left atrial strain changes in patients with breast cancer during anthracycline therapy. Int J Cardiol. 2021;330:238-244, doi: 10.1016/j.jijcard.2021.02.013, indexed in Pubmed: 33581179 . 